\title{
Representation of VR-Based Health Information for Smart City
}

\author{
Seo Eun Kim \\ Department of Oral Anatomy, School of Dentistry \& \\ Dental Research Institute \\ Seoul National University \\ Jongno-gu, Seoul, Republic of Korea \\ Chang Sup Park \\ Department of Computer Science \\ Dongduk Women's University \\ Seongbuk-gu, Seoul, Republic of Korea
}

\author{
Myeong Won Lee \\ Cheol Hee Jung \\ Department of Computer Science \\ The University of Suwon \\ Hwaseong, Gyenoggi-do, Republic of Korea \\ Ji Min Kim \\ Seung Pyo Lee \\ Department of Oral Anatomy, School of Dentistry \& \\ Dental Research Institute \\ Seoul National University \\ Jongno-gu, Seoul, Republic of Korea
}

\section{CCS CONCEPTS}

\begin{abstract}
A rich source of information relevant to smart cities and health care comes from sensors embedded in the environment, or ambient sensors. Strategically responding to sensed data helps healthcare would be smarter. By gaining real time access to this information, city services can respond promptly to urgent health needs and make decisions to avoid unhealthy situations. The emergence of virtual reality and ICT assisted urban planning effectively overcomes these complexities through visualization of big data. Web-based visualization technologies such as virtual reality (VR) present a highly detailed dynamic virtual environment. Also, there is continuously increasing demand for the effective organization of this diversified data, linkage with other data, and the visualization of data. In recent years, there has been a rapid increase in the development of health information systems motivated by legislation intended to protect patients' information and privacy and government interest in reducing the cost and improving the quality of healthcare.

In this paper, we investigate types of physiological content and the related sensors that can be included in a smart city healthcare system. We present a technical healthcare information model with XML in a standardized format for the implementation of a 3D virtual smart city as follows: Firstly, 3D virtual environment and healthcare information systems were confirmed to be dictated by sensor information parameters via XML. Secondly, real-time location, direction, and health data representation for 3D health management sensor data monitoring and real-time monitoring were conducted in XML. The identified key use case physiological sensor-based XML is helpful as it provides a standardized format for the implementation of a 3D virtual smart city. For implementation, X3D was used for visualizing an example health information system.
\end{abstract}

Permission to make digital or hard copies of all or part of this work for personal or classroom use is granted without fee provided that copies are not made or distributed for profit or commercial advantage and that copies bear this notice and the full citation on the first page. Copyrights for components of this work owned by others than ACM must be honored. Abstracting with credit is permitted. To copy otherwise, or republish, to post on servers or to redistribute to lists, requires prior specific permission and/or a fee. Request permissions from permissions@acm.org.

Web3D '20, November 9-13, 2020, Virtual Event, Republic of Korea

(c) 2020 Association for Computing Machinery.

ACM ISBN 978-1-4503-8169-7/20/11 . \$ \$15.00

https://doi.org/10.1145/3424616.3424698
- Human-centered computing $\rightarrow$ Information visualization.

\section{KEYWORDS}

Virtual reality, Smart city, Data visualization, Health information, Health information visualization

ACM Reference Format:

Seo Eun Kim, Myeong Won Lee, Cheol Hee Jung, Chang Sup Park, Ji Min Kim, and Seung Pyo Lee. 2020. Representation of VR-Based Health Information for Smart City. In The 25th International Conference on 3D Web Technology (Web3D '20), November 9-13, 2020, Virtual Event, Republic of Korea. ACM, New York, NY, USA, 7 pages. https://doi.org/10.1145/3424616.3424698

\section{INTRODUCTION}

Over the last two or three decades, technology, projects and initiatives related to smart city have been rapidly developed, affecting almost all aspects of urban life [Jing et al. 2019]. "Smart city" means a city that supports sustainable economic growth and a high quality of life using a smart platform of both traditional and modern information and communications technology (ICT) [Cook et al. 2018]. Its aim is to improve the quality of life of citizens via such things as transport infrastructures, communications infrastructures, energy savings, urban green area care, reduction of air pollution, and sustainable growth [Jamei et al. 2017]. Nowadays, as population continues to grow and human life expectancy increases, interest in healthcare monitoring is also increasing in order to bridge the gap between life expectancy and birth and healthy life years and to promote healthy living [Beltrán-Sánchez et al. 2015; Crimmins 2015]. To meet these goals, smart cities need to introduce new technologies and realize smart services that address the concerns and needs of citizens [Jamei et al. 2017] A human being has multiple different physiological signs that can be measured. It is possible to extract electro chemical, human biosignals and use them to better understand bodily health status and reaction to external factors [Dias and Paulo Silva Cunha 2018; Seshadri et al. 2019].

The emergence of virtual reality and ICT assisted urban planning allows these complexities to be effectively overcome through visualization of big data [Jamei et al. 2017]. Web-based visualization technologies such as VR present a very detailed dynamic virtual 
environment [MacEachren et al. 1999]. Data visualization has been simply represented as numbers which allows for emphasizing significant portions and conveying conclusions in a straightforward way. As the types of data generated in cities diversifies, the volume of data increases as does the rate of increase. As a result, there is increasing demand for ways to effectively organize this data, link it with other data, and visualize the data [Palanisamy and Thirunavukarasu 2019]. In the past few years, continuouslycollected data in ambient intelligent environments has been used to look for changes in health status and affect the design of home health interventions [Cook et al. 2018].

In recent years, there has been a rapid increase in the development of health information systems that is motivated by legislation intended to protect patients' information and privacy and government interest in reducing the cost and improving the quality of healthcare [of Medicine 2009]. Electronic health records allow healthcare institutions to store patient information more efficiently. Also, there is the increased spread of infection in recent years has become a global health problem. One of the most representative suspicious symptoms of infection is high fever. When this symptom occurs, the response process can be simulated with the assistance of virtual reality. In addition, work efficiency can be improved by visualizing characters in VR space which also promotes smooth communications services. A health information system provides fundamental smart health functionalities for the implementation of VR based smart city and can be extended using other health information. XML can be used to define an exchangeable data model for health information systems, and health information systems can be developed using XML descriptions [Brewton et al. 2012].

The aim of the present study was to investigate the types of physiological content and the related sensors that can be included in a smart city healthcare system. Based on this, a reference healthcare information model can then be defined with XML (Extensible Markup Language) in a standardized format for the implementation of a 3D virtual smart city.

\section{VR BASED HEALTH INFORMATION SYSTEM}

Maintenance of peoples' health and the smart city ability to respond quickly to health issues (such as accidents or disease outbreaks) are critical elements of smart city. In order for these two elements to be fulfilled, three processes are necessary. First, a health information system should be provided. Second, peoples' health should be monitored. Third, a system capable of storing each individual's customized information should be enacted. Through these processes, a constant evaluation of the health of the city's citizens is feasible and the city can determine if they are implementing the right functions in order to increase the health of its citizens.

\section{HEALTH SENSOR INFORMATION}

Representative physiological sign and related sensors can be presented as follows: blood glucose, blood pressure, electrocardiogram (ECG), heart rate, temperature, height (extensometer), weight, BMI, hyperlipidemia. Figure 2 shows the physiological signs for health information.

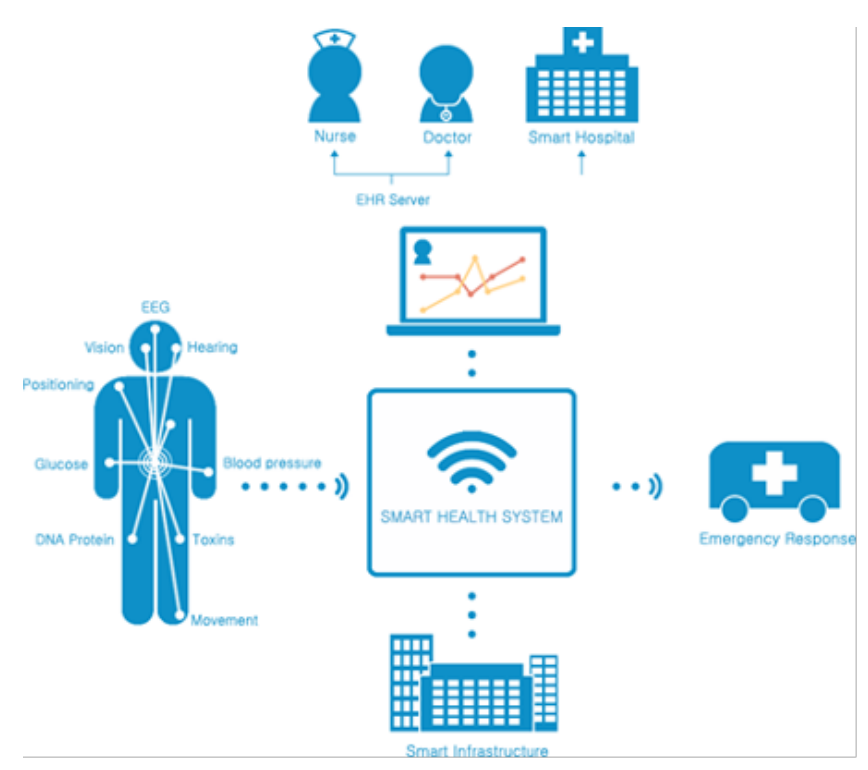

Figure 1: Smart health system in smart city.

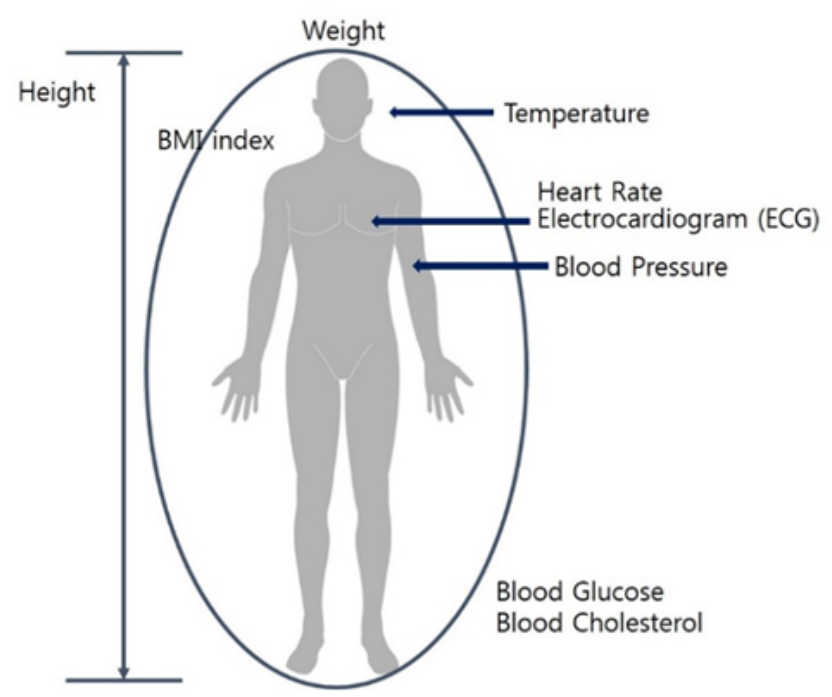

Figure 2: Physiological and chemical sensor types.

Each sensor type can be defined based on its physiological and chemical properties and the related devices that can be represented and simulated in a smart city. Physiological signs are classified as follows:

- Blood glucose is the concentration of blood sugar which is the primary source of energy for the body's cells. The glucose level is regulated in the human body and normally maintained between approximately $70 \mathrm{mg} / \mathrm{dL}$ and $150 \mathrm{mg} / \mathrm{dL}$ (4 mmol/L and $8 \mathrm{mmol} / \mathrm{L}$ ) [IEEE STD 10417 2015]. Glucose sensors on the current medical diagnosis market are mostly enzyme-based sensors, such as glucose oxidase or glucose 
dehydrogenase, and also utilize some optical and electrochemical methods [Wang and Lee 2015].

- Blood pressure $(\mathrm{mm} / \mathrm{Hg})$ refers to the cyclic fluid pressure against the walls of blood vessels. Non-invasive blood pressure measurement is typically performed at the brachial radial artery (arm or wrist) [IEEE STD 10407 2008]. Generally, the basic means of blood pressure measurement is divided into two main sensor types: analogue pressure sensor type and preelectronic pressure sensor type.

- Electrocardiogram (ECG) is a device with associated lead wires and electrodes that measures the electrical activity of the heart. It measures the electrical potential differences between electrodes placed on the person's body. Its sensor includes non-contact capacitive electrodes which are able to acquire ECG data without direct skin contact. However, motion artifacts can disrupt its precise measurement when compared to conventional electrodes [Dias and Paulo Silva Cunha 2018; IEEE STD 10406 2011].

- Heart rate is the number of heartbeats per unit of time (beats $/ \mathrm{m}$ ). The standard unit of measurement is beats per minute [IEEE STD 10406 2011]. Sensors that can measure the heart rate include pressure sensors and piezoelectric sensors. A pressure sensor measures atmospheric pressure, typically in the SI unit form of hecto-pascal $(1 \mathrm{hPa}=100 \mathrm{~Pa}$ = 1 millibar) [IEEE STD 2700-2014 2014].

- Body temperature $\left({ }^{\circ} \mathrm{C}\right)$ is the measurement of the core body temperature of a person [ISO/IEEE 10408 2010]. Body Temperature can be measured by piezoelectric sensor, resistance temperature detectors (RTDs), and thermistors.

- Human height or stature is the distance from the bottom of the feet to the top of the head when standing erect [Anibor et al. 2014]. Height (cm) can be detected by ultrasonic sensor. An ultrasonic sensor is a type of acoustic sensor of which there are three broad categories: transmitters, receivers, and transceivers [Babu et al. 2019].

- Human body weight refers to a person's mass or weight (kg). It is the measurement of weight without items located on the person using a weighing scale device [IEEE STD 10415 2008]. Weight can be measured by load cell. Load cell is a highly accurate transducer which provides the user with information generally not obtainable by other methods from the commercial perspective [Aravind et al. 2018].

- Body Mass Index (BMI) is defined as body mass divided by the square of body height and is universally expressed in units of $\mathrm{kg} / \mathrm{m} 2$ resulting from mass in kilograms and height in meters [Zierle-Ghosh and Jan 2019]. BMI is a measure for indicating an overweight or underweight condition of a person [IEEE STD 10415 2008].

- Hyperlipidemia is abnormally elevated levels of any or all lipids or lipoproteins in the blood [Karam 2017]. Blood cholesterol can be measured by electrochemical sensor $(\mathrm{mg} / \mathrm{dl})$.

The above parameters and their values captured from health devices in the real world should be able to be maintained in the virtual environment for monitoring the health information of a person. In order to manage all data related to health information, a data model was defined as in the following section.

\section{A DATA MODEL FOR HEALTH INFORMATION SYSTEMS}

This section describes XML definition for health information systems in 3D VR environments.

\subsection{Root Element}

The root element healthInfo consists of healthSensor elements of Physiological Sensor type. The XML schema definition is as follows:

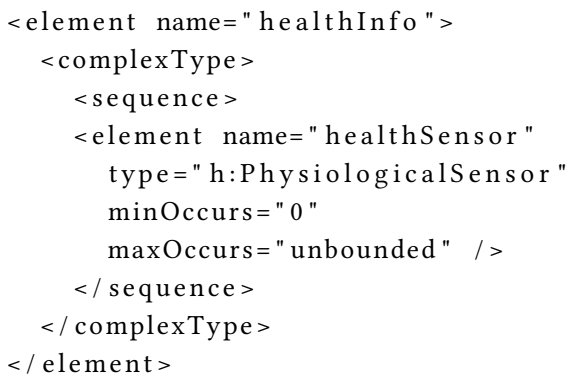

\subsection{Physiological Sensor Type}

Physiological sensor is an abstract super-type of all physiological sensor types, which include ID and type as follows:

- id: sensor identification

- type: sensor type which has one of nine values (BloodGlucose, BloodPressure, ECG, HeartRate, BodyTemperature, Height, Weight, BMI, BloodCholesterol)

The XML schema definition for the physiological sensor type is as follows:

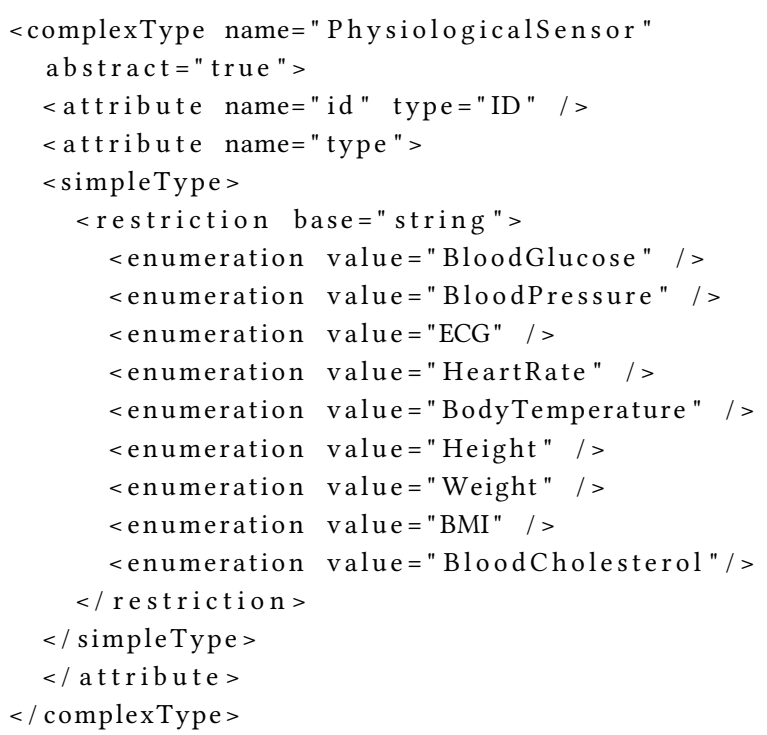

\subsection{Blood Glucose Sensor Type}

The blood glucose sensor type has two child elements representing fasting plasma glucose $(\mathrm{mg} / \mathrm{dL})$ and two-hour postload plasma glucose $(\mathrm{mg} / \mathrm{dL})$. The XML schema definition for the blood glucose sensor type is as follows: 


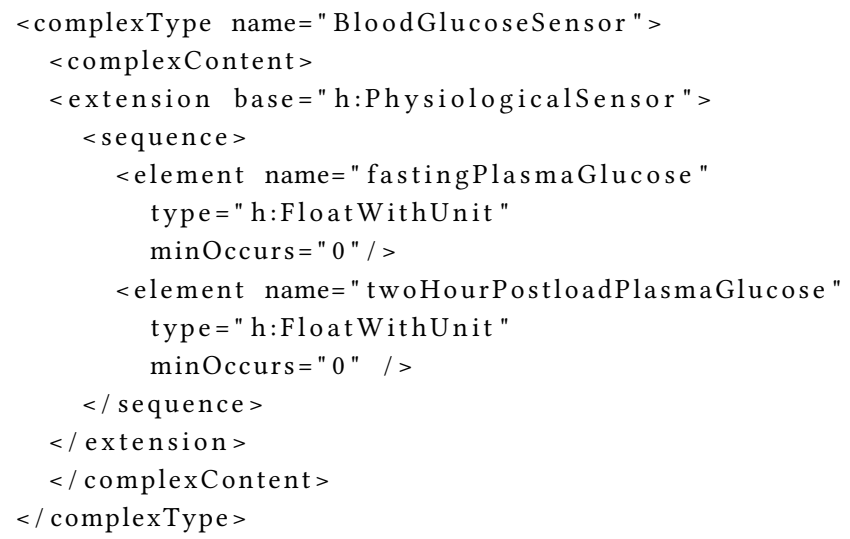

\subsection{Blood Pressure Sensor Type}

The blood pressure sensor type has two child elements representing systolic blood pressure $(\mathrm{mm} / \mathrm{Hg})$ and diastolic blood pressure (mm/Hg).

The XML schema definition for the blood pressure sensor type is as follows:

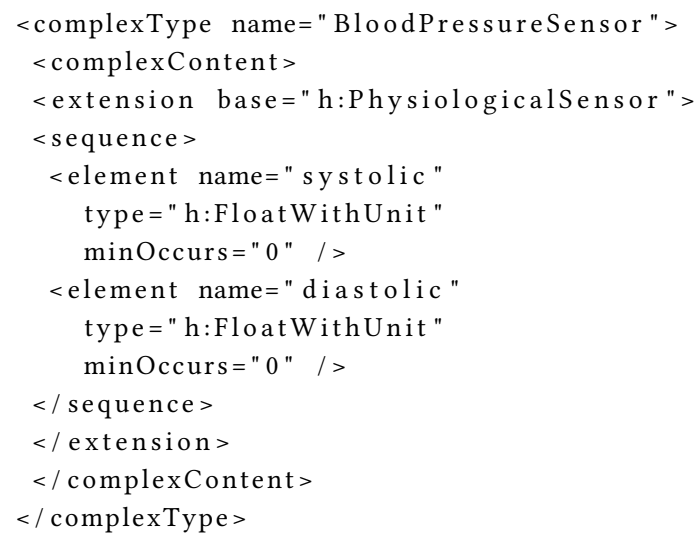

\subsection{ECG Sensor Type}

The ECG sensor type has a child element representing the electrical activity of the heart (waveform). A concrete data type for waveform should be defined.

The XML schema definition for the ECG sensor type is as follows:

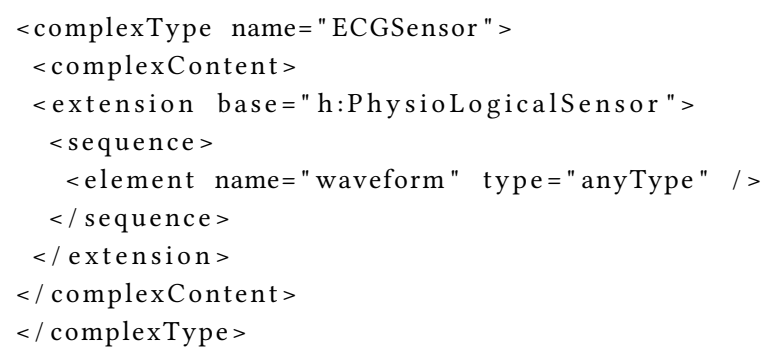

\subsection{Heart Rate Sensor Type}

The heart rate sensor type has a child element representing heartbeats (beats $/ \mathrm{m}$ ). The XML schema definition for heart rate sensor type is as follows:

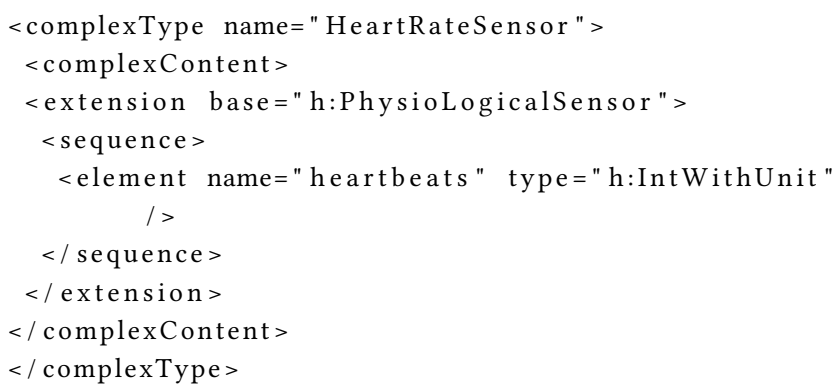

\subsection{Body Temperature Sensor Type}

The body temperature sensor type has a child element representing body temperature $\left({ }^{\circ} \mathrm{C}\right)$. The XML schema definition for the body temperature sensor type is as follows:

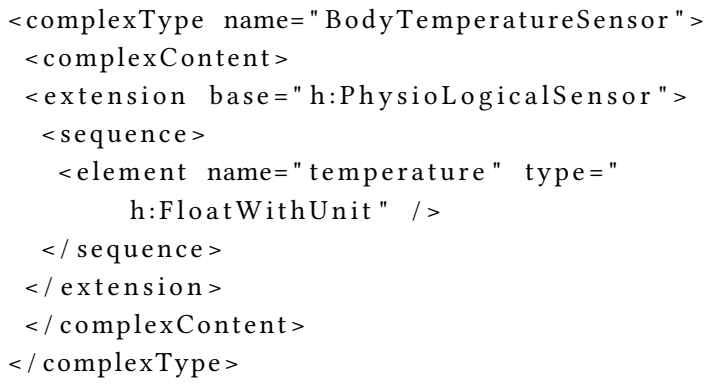

\subsection{Height Sensor Type}

The height sensor type has a child element representing height (cm). The XML schema definition for the height sensor type is as follows:

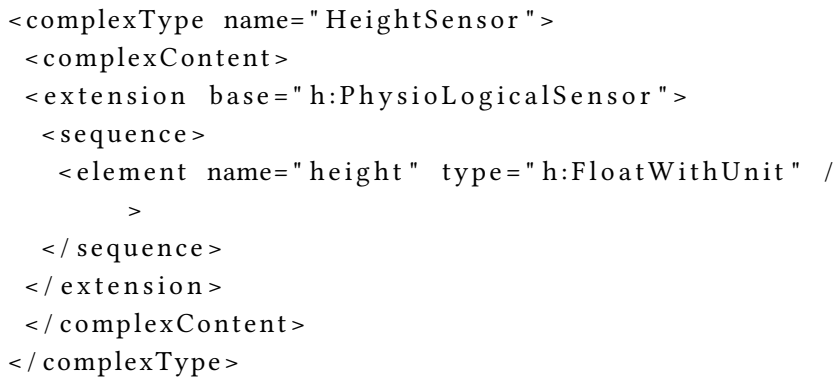

\subsection{Weight Sensor Type}

The weight sensor type has a child element representing weight (cm). The XML schema definition for the weight sensor type is as follows:

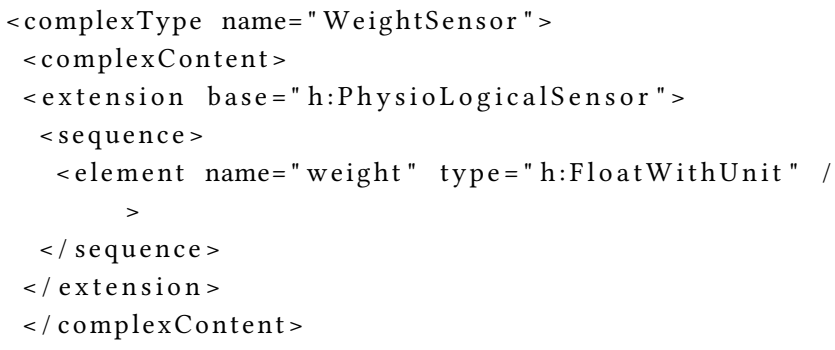


$</$ complexType $>$

\subsection{BMI Sensor Type}

The BMI sensor type has a child element representing BMI $(\mathrm{kg} / \mathrm{m} 2)$. The XML schema definition for the BMI sensor type is as follows:

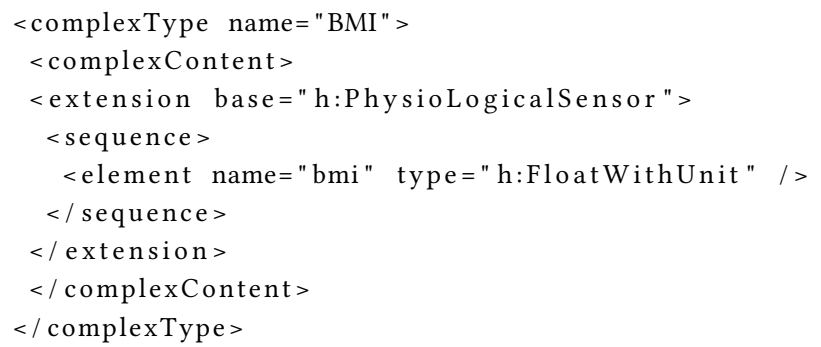

\subsection{Blood Cholesterol Sensor Type}

The Blood cholesterol sensor type has a child element representing LDL $(\mathrm{mg} / \mathrm{dL})$, HDL $(\mathrm{mg} / \mathrm{dL})$ and neutral fat $(\mathrm{mg} / \mathrm{dL})$. The XML schema definition for the blood cholesterol sensor type is as follows:

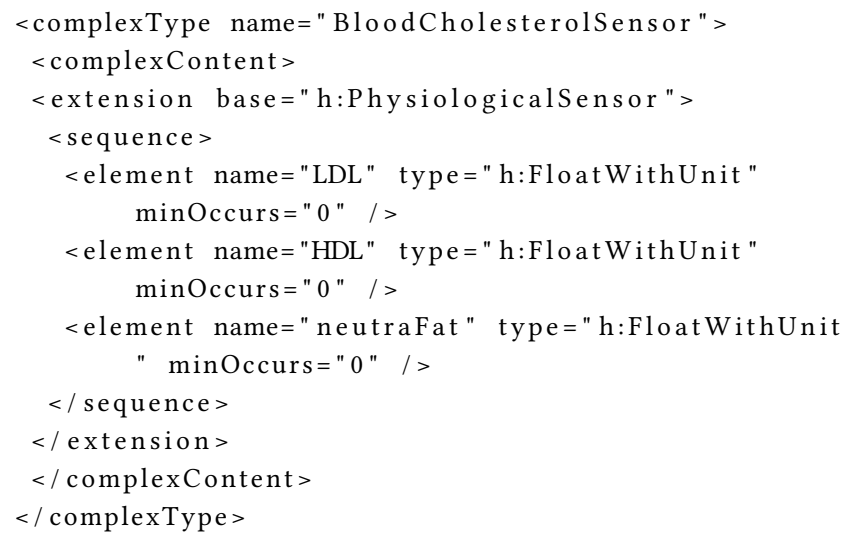

\subsection{Basic Data Types}

There are two data types that can be used to define data values for each element: IntWithUnit and FloatWithUnit. IntWithUnit represents an integer value with unit information. FloatWithUnit represents a float value with unit information.

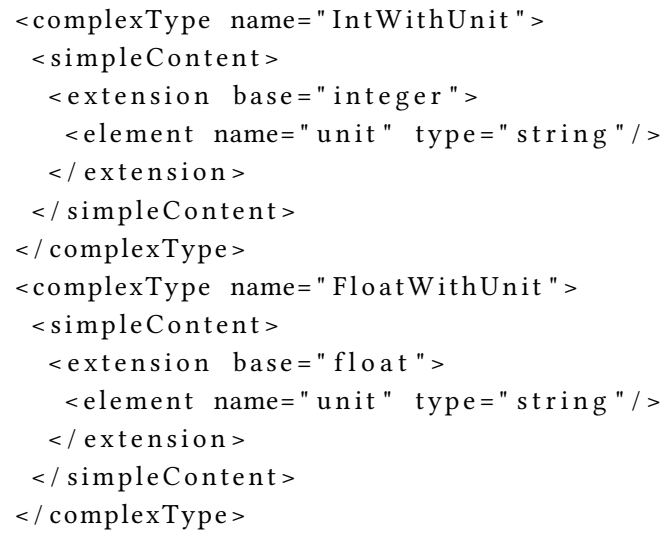

\subsection{Example XML document for health information}

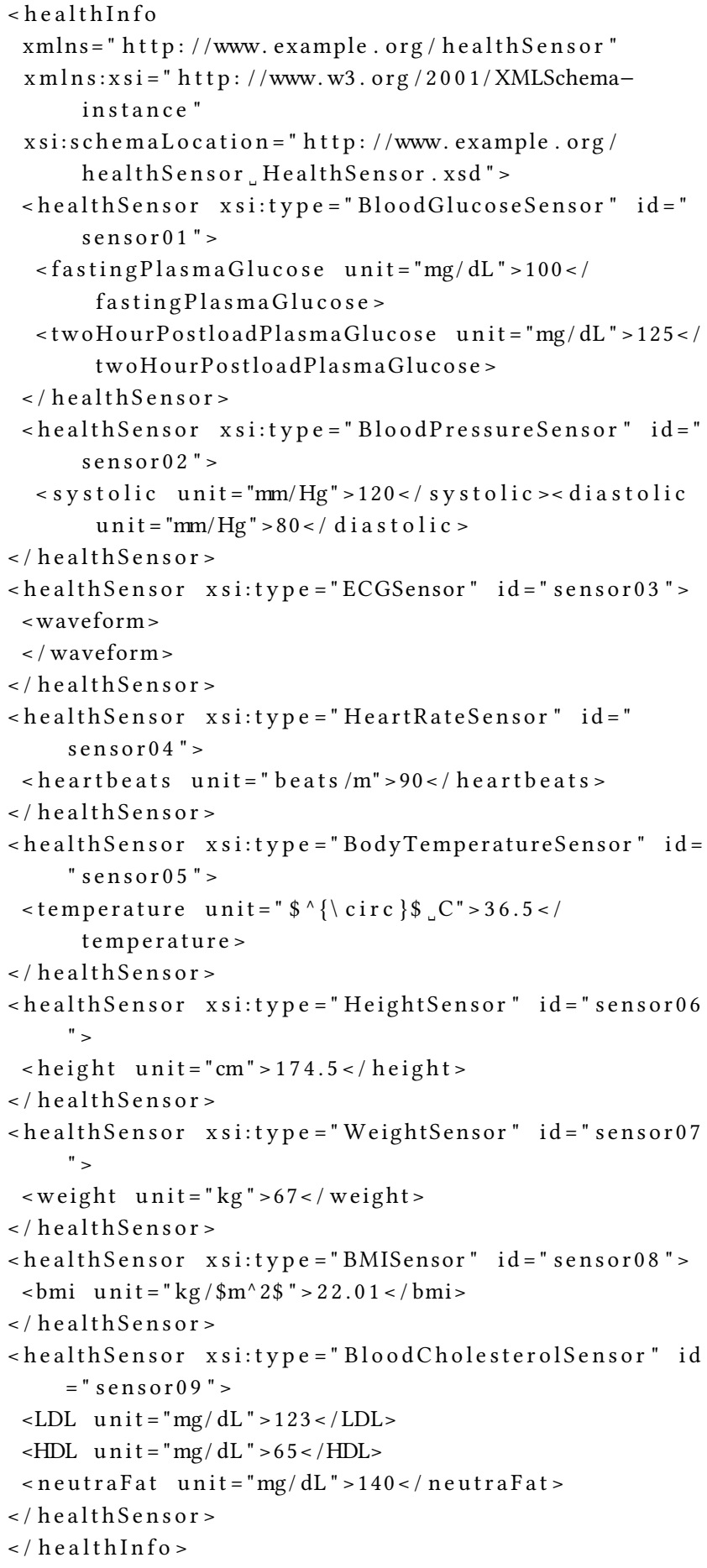

\section{AN IMPLEMENTATION EXAMPLE OF HEALTH INFORMATION SYSTEMS}

An example of a health information system has been implemented based on the data model defined in the previous sections. X3D was 
used for visualizing the system in the corresponding virtual world that represents a real world that includes people. The scenario for representing the health information in the virtual world that is simulating a real world is as follows: A person is walking around in the real world while their avatar is simultaneously walking around in the virtual world. The virtual world is a copy of the real world. This means that the person and the real world are represented in the virtual world at the same time. The health information of the person is obtained by their wearable health device sensors (for example, phone sensors or a health monitoring smart watch) and maintained in the virtual world. Blood pressure, heart rate, or temperature (as described in section 3) can be obtained from the devices. This health information can in turn be monitored and managed by using an X3D browser implemented for a desktop or a mobile phone.

In this example, we used X3D (ISO/IEC 19775) and HAnim (ISO/IEC 19774) for representing the 3D virtual world and the 3D avatar [HAnim Architecture 2019; HAnim Motion Data 2019; HAnim V3.3 2013]. The 3D environment is synchronized with the GPS information of the real environment, and the avatar representing the person is also being monitored using the person's GPS information in the real environment. X3D was used because it can represent GPS based 3D environments. The 3D environment corresponding to the real world is located in a GPS bounding area The 3D environment is synchronized with the avatar based on the GPS information. The health information is managed using the HAnim data representing the person. Because HAnim data can include attachments to the specific location of the avatar, sensor devices can be represented and operated in accordance with the GPS location of the person in the 3D environment. Because it can be difficult to obtain the change in the GPS location for a person in a given area, we used the geometric locations of the person after obtaining the GPS location of the area around the buildings.

Figure 3 shows the implementation of a 3D environment and an avatar using sensors for GPS, gyro, and heart rate in order to represent and manage human health information. C\# and Unity were used to implement the X3D based health information system. Sensor information should be synchronized with the avatar and the 3D environment. This means that the health information from sensors at each instance of time should be displayed in the $3 \mathrm{D}$ virtual environment as the person with sensors is walking around in the real world. Numeric values such as for GPS, steps taken, and heart rate are captured by sensors and displayed in the 3D virtual environment. Figure 4 shows communication between avatars in the $3 \mathrm{D}$ virtual environment as their corresponding persons are communicating in the real world.

\section{CONCLUSIONS}

In this paper, we classified health information parameters that can be obtained and monitored in a VR based smart city environment and we defined an XML data model for representing health information in a virtual environment. In order to monitor health information using health devices, the health information should be able to be represented by synchronizing the real world and the person with the corresponding virtual environment and avatar This means that the virtual environment should be able to be represented with geographically correct locations and that the location

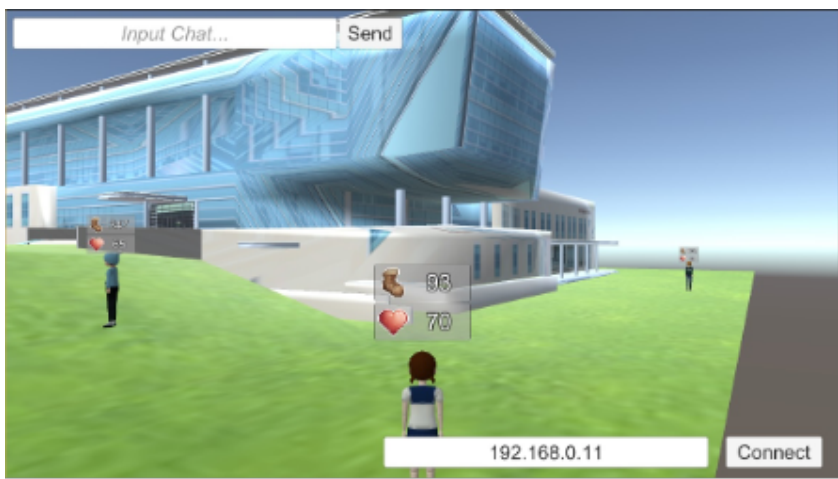

Figure 3: Synchronization of persons in the real world with their avatars in the 3D virtual environment.

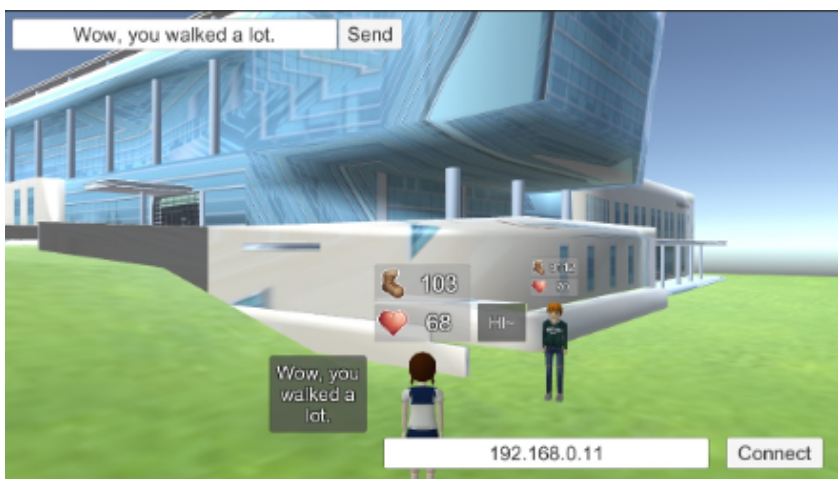

Figure 4: Communication between avatars in the 3D virtual world as their corresponding persons communication in the real world.

of the avatar should be able to be traced and mapped in the virtual environment using sensor device information from the real world.

In order to synchronize the virtual environment and the avatar correctly with the real environment and the person, 3D data composing the virtual environment and avatar should be able to be detected semantically. Using X3D and HAnim, the virtual environment and the avatar can be synchronized with the real environment and the person. The data model for the health information is defined with XML. This model can be integrated with X3D and HAnim to manage and control health information in the synchronized environment with the avatar in order to monitor the health information for the person in the real world.

\section{ACKNOWLEDGMENTS}

This work was supported by the Standard Technology Development and Spread Program of KATS/KEIT(20001634, Development of VR/AR based smart city visualization system standards), and by MSIT, Korea, under the ITRC(Information Technology Research Center) support program (IITP-2020-2016-0-00312) supervised by the IITP. 


\section{REFERENCES}

E. Anibor, E. Ogbor-Omorie, and A. Nwagbara. 2014. The use of arm span to estimate height among the Urhobos in Delta state of Nigeria. African fournal of Cellular Pathology 3, 10 (2014), 16-19. https://doi.org/10.5897/AJCPATH14.016

R. Aravind, Easwaran Arunkumar, K HarisudhanR, G KaranRaj, and G. Udhayakumar. 2018. Load Cell based Fuel Level Measurement using Arduino Uno Microcontroller. International fournal for Advance Research and Development 3 (2018), 159-164.

T. Babu, S. Ashwin, Mukul Naidu, C. Muthukumaaran, and C. Ravi Raghavan. 2019 Sleep Detection and Alert System for Automobiles. In Advances in Manufacturing Technology, Somashekhar S. Hiremath, N. Siva Shanmugam, and B. R. Ramesh Bapu (Eds.). Springer Singapore, Singapore, 113-118.

Hiram Beltrán-Sánchez, Samir Soneji, and Eileen M. Crimmins. 2015. Past Present, and Future of Healthy Life Expectancy. Cold Spring Harbor Perspectives in Medicine 5, 11 (2015). https://doi.org/10.1101/cshperspect.a025957 arXiv:http://perspectivesinmedicine.cshlp.org/content/5/11/a025957.full.pdf + html J. Brewton, X. Yuan, and Francis Akowuah. 2012. XML in Health Information Systems.

D. J. Cook, G. Duncan, G. Sprint, and R. L. Fritz. 2018. Using Smart City Technology to Make Healthcare Smarter. Proc. IEEE 106, 4 (2018), 708-722.

Eileen M. Crimmins. 2015. Lifespan and Healthspan: Past, Present, and Promise. The Gerontologist 55, 6 (11 2015), 901-911. https://doi org/10.1093/geront/gnv130 arXiv:https://academic.oup.com/gerontologist/articlepdf/55/6/901/8081968/gnv130.pdf

Duarte Dias and João Paulo Silva Cunha. 2018. Wearable Health Devices-Vital Sign Monitoring, Systems and Technologies. Sensors 18, 8 (Jul 2018), 2414. https: //doi.org/10.3390/s18082414

HAnim Architecture 2019. V2.0 Humanoid Animation (HAnim) Part 1: Architecture https://www.web3d.org/content/hanim-architecture-v2

HAnim Motion Data 2019. V2.0 Humanoid Animation (HAnim) Part 2: Motion data animation. https://www.web3d.org/documents/specifications/19774-2/V2.0/ MotionDataAnimation/MotionDataAnimation.html

HAnim V3.3 2013. V3.3 Extensible 3D (X3D). https://www.web3d.org/standards/ version/V3.3

2011. Health informatics-Personal health device communication Part 10406: Device specialization-Basic electrocardiograph (ECG) (1- to 3-lead ECG). IEEE STD 1107310406-2011 (2011), 1-73.

2008. Health informatics-Personal health device communication Part 10407: Device specialization - Blood pressure monitor. IEEE STD 11073-10407-2008 (2008), c1-41.
2008. IEEE Health Informatics - Personal Health Device Communication - Device Specialization - Weighing Scale. IEEE Std 11073-10415-2008 (2008), 1-42.

2015. IEEE Health informatics - Personal health device communication Part 10417: Device Specialization - Glucose Meter. IEEE Std 11073-10417-2015 (Revision of IEEE Std 11073-10417-2011) (2015), 1-78.

2014. IEEE Standard for Sensor Performance Parameter Definitions. IEEE STD 2700-2014 (2014), 1-69.

2010. ISO/IEEE Health informatics Personal health device communication Part 10408: Device specialization Thermometer. ISO/IEEE 11073-10408:2010(E) (2010), 1-48.

Elmira Jamei, Michael Mortimer, Mehdi Seyedmahmoudian, Ben Horan, and Alex Stojcevski. 2017. Investigating the Role of Virtual Reality in Planning for Sustainable Smart Cities. Sustainability 9, 11 (Nov 2017), 2006. https://doi.org/10.3390/ su9112006

Changfeng Jing, Mingyi Du, Songnian Li, and Siyuan Liu. 2019. Geospatial Dashboards for Monitoring Smart City Performance. Sustainability 11, 20 (Oct 2019), 5648. https://doi.org/10.3390/su11205648

Isam Karam. 2017. Hyperlipidemia Background and Progress. SM Atherosclerosis fournal Volume 1 (08 2017), 1-8.

Alan M. MacEachren, Robert Edsall, Daniel Haug, Ryan Baxter, George Otto, Raymon Masters, Sven Fuhrmann, and Liujian Qian. 1999. Virtual Environments for Geographic Visualization: Potential and Challenges. In Proceedings of the 1999 Workshop on New Paradigms in Information Visualization and Manipulation in Conjunction with the Eighth ACM Internation Conference on Information and Knowledge Management (Kansas City, Missouri, USA) (NPIVM '99). Association for Computing Machinery, New York, NY, USA, 35-40. https://doi.org/10.1145/331770.331781

Institute of Medicine. 2009. Beyond the HIPAA Privacy Rule: Enhancing Privacy, Improving Health Through Research. The National Academies Press, Washington, DC. https://doi.org/10.17226/12458

Venketesh Palanisamy and Ramkumar Thirunavukarasu. 2019. Implications of big data analytics in developing healthcare frameworks - A review. Fournal of King Saud University - Computer and Information Sciences 31, 4 (2019), 415 - 425. https: //doi.org/10.1016/j.jksuci.2017.12.007

D. Seshadri, R. Li, J. Voos, J. Rowbottom, C. Alfes, C. Zorman, and C. Drummond. 2019. Wearable sensors for monitoring the physiological and biochemical profile of the athlete. npj Digital Medicine 2, 72 (2019). https://doi.org/10.1038/s41746-019-0150-9

Hui-Chen Wang and An-Rong Lee. 2015. Recent developments in blood glucose sensors. Journal of Food and Drug Analysis 23, 2 (2015), 191 - 200. https://doi.org/10.1016/j. jfda.2014.12.001

A. Zierle-Ghosh and A. Jan. 2019. Physiology, Body Mass Index (BMI) 